Supporting Information

\title{
Direct Fabrication of a Moisture-Driven Power Generator by Laser- Induced Graphitization with a Gradual Defocusing Method
}

Sanghee Lee, ${ }^{\mathrm{a}}$ Hansol Jang, ${ }^{\mathrm{a}}$ Hansol Lee, ${ }^{\mathrm{a}}$ Dongho Yoon $^{\mathrm{b}}$ and Sangmin Jeon ${ }^{\mathrm{a} *}$

\footnotetext{
a Department of Chemical Engineering, Pohang University of Science and Technology (POSTECH), 77 Cheongam-Ro, Pohang, Gyeongbuk, Republic of Korea

${ }^{\mathrm{b}}$ R\&D Center, KUK IL PAPER MFG Co., LTD., Baekok-daero 563, Cheoin-gu, Yongin, Republic of Korea

* Corresponding Author

*E-mail: jeons@postech.ac.kr
}

Number of Pages: 3

Number of Figures: 2

Number of Tables: 0 


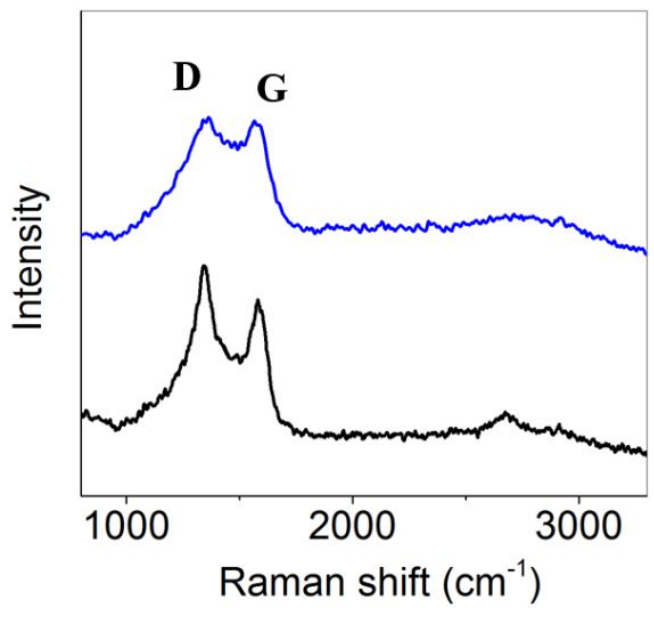

Figure S1. Raman spectra of section $A$ (blue) and $C$ (black) of GCL in Figure 3a. The $G$ band of section $C$ is sharper than that of section $A$, indicating that more graphitization occurred in section $C$. 


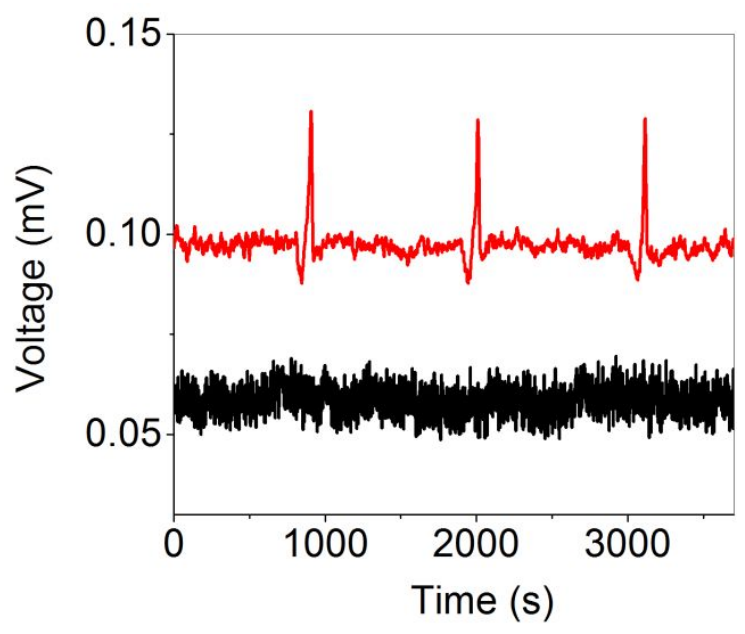

Figure S2. Voltage outputs from GCLs fabricated without substrate inclination. The laser beam defocus distance varied from $0 \mathrm{~mm}$ (black, focal plane) to $2.7 \mathrm{~mm}$ (red).

A cellulose substrate was laser irradiated without substrate inclination to obtain GCLs with uniform $\mathrm{O} / \mathrm{C}$ values. Two uniform GCL samples were fabricated at different defocus distances: $0 \mathrm{~mm}$ (GCL-A) and $2.7 \mathrm{~mm}$ (GCL-B). Figure S2 shows that the voltage outputs from GCL-A and GCL-B were nearly 0 and $<0.03 \mathrm{mV}(230 \mathrm{mV}$ from the GCL fabricated at a tilt angle of $56^{\circ}$ ), respectively, when exposed to $70 \% \mathrm{RH}$, indicating that the $\mathrm{O} / \mathrm{C}$ gradient was critical for generating electricity. The voltage output from GCL-A was smaller than from GCL-B due to the lower O/C of GCL-A. 